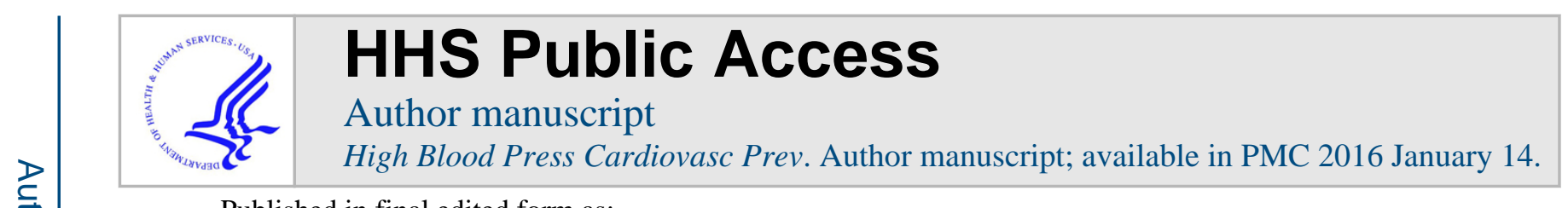

Published in final edited form as:

High Blood Press Cardiovasc Prev. 2014 December ; 21(4): 269-274. doi:10.1007/s40292-014-0064-1.

\title{
Antihypertensive Medications and Diastolic Dysfunction Progression in an African American Population
}

\author{
Heather M. Prendergast, \\ Department of Emergency Medicine, University of IIIlinois Hospital \& Health Sciences System, \\ 808 S. Wood Rm 471-H, Chicago, IL 60612, USA
}

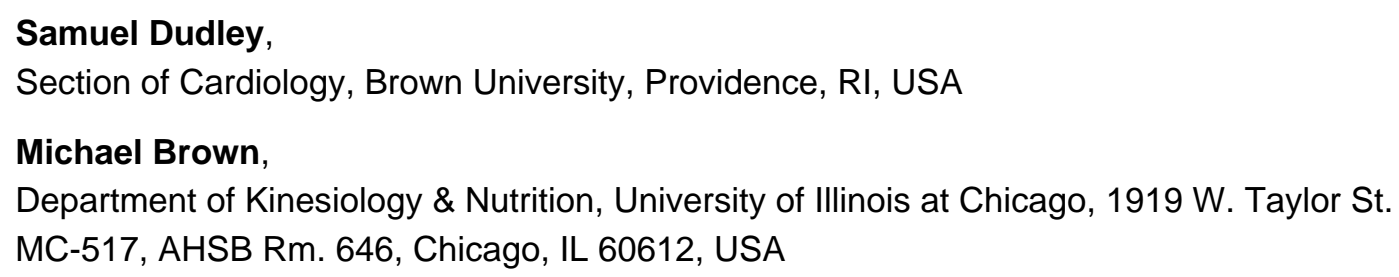

\section{Martha Daviglus,}

Institute for Minority Health, University of Illinois at Chicago, 1819 W. Polk St, Suite 246, Chicago, IL 60612, USA

\section{John Kane,}

Rush Medical Center, Chicago, USA

\section{E. Bradshaw Bunney,}

Department of Emergency Medicine, University of Illlinois Hospital \& Health Sciences System, 808 S. Wood Rm 471-H, Chicago, IL 60612, USA

\section{Jared Marcucci, and}

Department of Emergency Medicine, University of Illlinois Hospital \& Health Sciences System, 808 S. Wood Rm 471-H, Chicago, IL 60612, USA

\section{Rasheed Sanyaolu}

Department of Emergency Medicine, University of IIllinois Hospital \& Health Sciences System, 808 S. Wood Rm 471-H, Chicago, IL 60612, USA

Heather M. Prendergast: hprender.heather@gmail.com, hprender@uic.edu; Samuel Dudley: samuel_dudley@brown.edu; Michael Brown: brownmd@uic.edu; Martha Daviglus: daviglus@uic.edu; John Kane: john_kane@rush.edu; E. Bradshaw Bunney: brad.bunney@gmail.com; Jared Marcucci: jaredmarcucci@gmail.com; Rasheed Sanyaolu: rsanya3@uic.edu

\section{Abstract}

Background-There have been no studies examining the association between antihypertensive medications and progression of diastolic dysfunction. The purpose of this study is to determine whether there is an association between class of antihypertensive medication and diastolic dysfunction progression in an African American population.

Methods-A retrospective cohort study of African American patients with at least two echocardiograms demonstrating diastolic dysfunction drawn from an echocardiogram database at 
an academic medical center. The main outcome measures were change in diastolic function grade as a function of time and association with hypertension medication classification.

Results-There were 96 African American patients in the database with 2 or more echocardiograms demonstrating diastolic dysfunction; representing 302 echocardiograms. The mean time between echocardiograms was 2.6 years. The mean age was $64.2( \pm 10.1)$ years, $78 \%$ were women, and mean BMI $31.2( \pm 7.4) \mathrm{kg} / \mathrm{m}^{2}$. The majority of subjects had Grade I diastolic dysfunction at their initial examination $[\mathrm{N}=87(90.6 \%)]$. Approximately $22.9 \%(\mathrm{n}=22)$ of the study cohort demonstrated diastolic dysfunction progression. In multivariate analysis use of calcium channel blockers (CCB) was protective against diastolic dysfunction progression (OR for CCB users vs. non-users $0.28,95 \%$ confidence interval, $0.09-0.90, \mathrm{p}<0.05$ ).

Conclusions-These findings suggest that use of calcium channel blockers may have a protective effect against progression of diastolic dysfunction among African American patients. Further studies are required to confirm these findings and identify specific factors that can mediate disease progression among African American individuals with hypertension, who face substantial risk of complications such as diastolic heart failure.

\section{Keywords}

Diastolic dysfunction; Progression; Antihypertensive medications; African-American

\section{Introduction}

The prevalence of hypertension in African Americans in the United States is among the highest worldwide with a prevalence rate of $43 \%$ in men and $45.7 \%$ in women [1]. Compared to non-Hispanic whites, African Americans experience earlier onset of hypertension, higher rates of cardiovascular complications such as stroke and heart failure, and 4-5 times higher all-cause mortality [1]. It is well established that hypertension is a major contributor to the development of heart failure, particular diastolic heart failure. Treatment is one of the keys to preventing the development of diastolic heart failure [2]. In the United States, African Americans have the highest prevalence of heart failure present with heart failure at younger ages, and are less likely to have coronary artery disease as the primary etiology [3]. Generally, it is thought that asymptomatic diastolic dysfunction precedes overt heart failure, which may represent both an ideal time for intervention and a potential marker for disease progression [4]. Several different classes of antihypertensive agents have demonstrated effectiveness in the management of hypertension and prevention of secondary cardiovascular complications such as heart failure, including angiotensinconverting enzyme inhibitors and angiotensin receptor blockers [5]. However, a recent study found lower responsiveness in maintaining optimal blood pressure control to monotherapy with ACE inhibitors and angiotensin receptor blockers in an African American population, suggesting that the optimal choice of antihypertensive drugs in African American patients for prevention of cardiovascular complications such as heart failure may be different than that observed in non-minority populations [1]. The 2014 evidence-based guideline for the management of high blood pressure in adults (JNC-8) suggest in the black hypertensive population a calcium channel blocker or thiazide-type diuretic is recommended as initial therapy [6]. Recent studies highlight the importance of tailoring the antihypertensive 
selection to both symptomatology and comorbidities [7]. This study aims to determine whether class of antihypertensive medication used is associated with diastolic dysfunction progression among African American individuals with evidence of diastolic dysfunction.

\section{Methods}

A retrospective cohort was established by selecting patients from the echocardiogram database-including both inpatient and outpatient diagnostic studies—and the Electronic Health Record (EHR) system at the University of Illinois Healthcare System. All individuals with at least two echocardiograms positive for diastolic dysfunction during the study period (2006-2012) were selected; Since the inclusion criteria were based upon presence of diastolic dysfunction on 2 or more echocardiograms during the study period, individuals with no evidence of diastolic dysfunction at time zero but who developed diastolic dysfunction on subsequent echocardiograms were included in the study cohort.

Echocardiogram data was cross-referenced by medical record number to existing health records. The University of Illinois Institutional Review Board approved the study.

\subsection{Participants}

A total of 154 participants were selected for inclusion in the study. Among the original study cohort, the racial/ethnic classifications were as follows: $62 \%$ African American, $20 \%$ Latino, $12 \%$ Caucasian, $7 \%$ Asian, and $1 \%$ Native American. The current analyses are based on data from African Americans patients only, who represented the majority of study participants $(\mathrm{N}=96 ; 62 \%)$.

Inclusion Criteria: 2 or more echocardiograms reporting diastolic dysfunction at anytime during the review period were eligible for inclusion in the study cohort to allow examination of progression rates $(\mathrm{N}=96)$. Patients were not excluded on the basis of echocardiogram indication, inpatient or outpatient status, or the presence or absence of cardiovascular risk factors (hypertension, diabetes, tobacco use, and coronary artery disease). A random sample of $10 \%$ of patients in the database who underwent only one echocardiogram was drawn to assess potential selection bias $(\mathrm{N}=240)$. Compared to persons with only 1 echocardiogram, patients included in this study had a higher proportion of women (78 vs. 52\%), smokers (71 vs. $38 \%$ ), and were older (51 vs. $2 \%$ age > 70). However the rates of diabetes were similar (62 vs. $62 \%$ ). Cohort Study Variables: Baseline demographic characteristics, hypertension medication classification, primary indication for the echocardiogram, co morbidities (past medical history), clinical data (blood pressure, blood urea nitrogen, creatinine, and hemoglobin levels) and echocardiography parameters (left atrial size (LA), diastolic dysfunction grade, and left ventricular hypertrophy $(\mathrm{LVH})$ were recorded.

\subsection{Echocardiograms}

Diastolic function was assessed in a standardized method, and in accordance to published guidelines at the time of the test using standard spectral Doppler and Tissue Doppler. All echocardiogram reports for the study cohort were available for review. Individual echocardiograms were interpreted by the clinical cardiologist assigned to the echocardiogram lab at the time of the diagnostic study. Board certified cardiologists 
interpreted all echocardiograms and echocardiogram readings were subjected to departmental quality review standards and procedures. Diastolic function was determined by estimating LA pressure using E/e' ratio. Diastolic dysfunction considered to be present if E/e '(septal) was greater than or equal to 15 , if $\mathrm{E} / \mathrm{e}^{\prime}$ (lateral) was greater than or equal to 12 , if septal $\mathrm{e}^{\prime}$ was less than $8 \mathrm{~cm} / \mathrm{sec}$, if lateral $\mathrm{e}^{\prime}$ was less than $10 \mathrm{~cm} / \mathrm{s}$. Diastolic function was considered to be absent if $\mathrm{E} / \mathrm{e}^{\prime}$ (septal or lateral) was less than or equal to 8 . For patients with E/e' between 9 and 12, the patient was considered to have elevated LAP if LA diameter was greater than $46 \mathrm{~mm}$ in the short axis [8]. Grading of diastolic dysfunction: Once diastolic dysfunction was determined, the E/A ratio was used to determine the grade of diastolic dysfunction. If E/A ratio was less than 0.8 , then the patient was considered to have grade I diastolic dysfunction. If E/A was between 0.8 and 1.5, then the patient was considered to have grade II diastolic dysfunction. If E/A was greater than 1.5, the patient was considered to have grade III diastolic dysfunction. [17] Diastolic dysfunction was graded on a 3 point ordinal scale; (1) mild diastolic dysfunction (Grade I, impaired relaxation); (2) moderate diastolic dysfunction (Grade II, pseudo-normal); and (3) severe diastolic dysfunction (Grade III, restrictive) [8].

Left ventricular hypertrophy (LVH), defined as an increase in the mass of the left ventricle, was estimated from LV measurements using the American Society of Echocardiography (ASE) guidelines [9].

Due to the retrospective nature of the study no intra and inter-observer reproducibility of echocardiographic measurements were available.

\subsection{Evaluation of Diastolic Dysfunction Progression}

Every patient's diastolic dysfunction grade was evaluated at first assessment. At subsequent visits diastolic dysfunction grades were monitored. If it any point during observation a patient's grade increased from first assessment along the spectrum of grades $(1,2,3)$, then a patient was considered to have progressed, even if that patient's diastolic dysfunction later regressed. Within four years of first assessment 20 (20.8\%) of the 96 patients had progressed and within five years $22(22.9 \%)$ patients had progressed.

\subsection{Statistical Analysis}

Descriptive characteristics of study participants at time of the initial echocardiogram, including demographics, physical measures, medication use, medical history, and echocardiogram parameters, were computed by presence or absence of diastolic dysfunction progression. For continuous variables, $t$ tests or Wilcoxon two-sample tests were used to test significance of difference between the 2 groups, as appropriate; for categorical variables, Pearson's chi-square test of Fisher's exact test, were used as appropriate.

Covariates considered for univariate analysis included age, gender, follow-up time (years), body mass index (BMI), systolic and diastolic blood pressures, blood urea nitrogen (BUN), creatinine, hemoglobin, hypertension medication classification (i.e. angiotensin converting enzyme inhibitors, angiotensin II receptor blockers, beta blockers, calcium channel blockers, 
diuretics, vasodilators, and central agonists), presence of diabetes, tobacco use, left ventricular hypertrophy (LVH) and left atrial (LA) size.

Covariates considered for multivariate analysis included hypertension medication classification (i.e. angiotensin converting enzyme inhibitors, angiotensin II receptor blockers, beta blockers, calcium channel blockers, diuretics, vasodilators, and central agonists) age, gender, body mass index (BMI), presence of diabetes, tobacco use, left ventricular hypertrophy (LVH) and left atrial (LA) size. Covariates were selected based upon the reviewed medical literature [6, 7]. Backward stepwise elimination logistic regression analysis was performed to examine the association of risk factors with progression of diastolic dysfunction.. Patients' time to observation of progression, or if no progression occurred, total time of observation, was controlled for at each step. Both Likelihood-ratio and Hosmer-Lemeshow tests were used to assess model fit.

\section{Results}

\subsection{Cohort Characteristics}

This study included data from 96 African American patients representing 302 echocardiograms and included both inpatient and outpatient studies. The primary indication for echocardiogram was chest pain followed by dyspnea. The study cohort was observed over a period ranging from 1.0 to 5.5 years per patient with an average duration of 2.6 years between repeat echocardiograms. Each patient had a mean (SD) of 4.2 (1.3) echocardiograms (range 3-10) during that time. At the time of the initial echocardiogram, the mean age of the 96 study participants was 64.2 (SD 10.1) years, mean BMI was 31.2 (SD 7.1) $\mathrm{kg} / \mathrm{m}^{2}$, and the majority of participants were women $(75,78.1 \%)$ (Table 1).

\subsection{Covariate Risk Factor Analysis}

In univariate analysis, risk factors were evaluated by progression status. Continuous variables were analyzed via t-tests comparing the means between the subset of the cohort whose diastolic dysfunction progressed and those who did not. Dichotomous variables were analyzed via Pearson's chi-square test for the proportions of those patients who progressed and those that did not and their diastolic dysfunction progression status (Table 2).

\subsection{Covariates Associated with Progression (Worsening) Diastolic Function}

Twelve risk factors were considered in the analysis: age, body mass index, diabetes, tobacco use, use of Beta Blockers, ACE Inhibitors, Angiotensin receptor blockers, calcium channel blockers, diuretics, LVH and LA Size. A multivariate logistic regression was performed to assess significant risk factors upon controlling for other risk factors including the differences in follow-up time between those with and without progression. In multivariate modeling, baseline use of CCB was the only variable significant at $\mathrm{p}<0.05$. Its effect is protective, with baseline users of CCB having an odds ratio of 0.28 (95\% confidence interval 0.09 , 0.90 ) relative to non-users (Table 3 ). 


\section{Discussion}

In this analysis, we found that the progression rates of diastolic dysfunction were significantly lower in those African Americans taking calcium channel blockers (CCB) as compared to other hypertension medications. Studies have shown that African Americans may differ in their BP response to particular classes of antihypertensive medications [10]. The effectiveness of CCBs in Africans Americans as monotherapy if blood pressures are $\leq 1 \mathrm{~mm} \mathrm{Hg}$ above target levels have been established and are part of the published guidelines from the International Society on Hypertension in Blacks [10]. Based upon the primary mechanism of action of calcium channel blockers the racial/ethnic variations in response to these agents in hypertensive minority patients are plausible. Recent studies have demonstrated the integral role for endothelial nitric oxide synthase/nitric oxide (eNOS/NO) cascade system in the pharmacological actions of selected calcium channel antagonist activity and the often suppressed nitric oxide levels in minorities [11]. Calcium channel blockade causes up-regulation of endothelial nitric oxide synthase expression leading to greater relaxation in the vasculature $[12,13]$. It has previously been established that hypertensive African Americans tend to have decreased nitric oxide bioavailability [14]. This is the first study to demonstrate a potential role of CCBs in secondary cardiovascular prevention in an African American population with evidence of diastolic dysfunction. One of the primary goals of antihypertensive therapy is to reduce cardiovascular events and data suggest that blood pressure management is the primary determinant of cardiovascular risk reduction which would support our study findings of lower progression rates with CCBs in African Americans [10]. A recent secondary analysis involving patients with known CAD, the Comparison of Amlodipine versus Enalapril to Limit Occurrences of Thrombosis (CAMELOT) trial, found that compared to placebo amlodipine resulted in fewer ischemic events; however this study involved a predominately non-minority patient population [15].

Although the prevalence of heart failure with preserved ejection fraction (HFpEF) is increasing and now accounts for $50 \%$ of all cases of heart failure, there are still many unresolved issues regarding treatment $[2,16]$. The primary pathophysiological mechanisms underlying HFPEF are fibrosis and reduced ventricular compliance [2]. Hypertension has been shown to be the most significant risk factor for the development of left ventricular diastolic dysfunction [17]. A recent meta-analysis of randomized controlled studies evaluating the effects of antihypertensive treatment in patients over 65 years of age on cardiovascular mortality and development of heart failure found that among different antihypertensive regimens with equal BP reduction, there were similar effects on cardiovascular outcomes [18]. In patients with evidence of LV systolic dysfunction the clear benefits of broad neurohormonal blockade with agents such as combined use of ACE inhibitors or ARBs, beta blockers, and in certain cases aldosterone antagonists has been established [19]. However for individuals with diastolic dysfunction these same improvements have not been consistently observed particular among different racial/ethnic groups [20]. The Anti-hypertensive and Lipid-Lowering Treatment to Prevent Heart Attack (ALLHAT) Trial examined the efficacy of different classes of antihypertensive medications and found that the ACE inhibitor lisinopril was less effective than thiazide direutics and CCBs in decreasing blood pressure and preventing major clinical outcomes including heart 
failure in African American participants compared to other racial groups [20]. Additional studies are needed to prospectively validate these findings as well as our study findings in other minority populations.

\section{Limitations}

Limitations of this study include a retrospective study design, the variability in the timing of repeated echocardiograms and the lack of inter observer reproducibility of echocardiographic measurements. The small number of subjects that had more than one echocardiogram, the high proportion of women, and the older age of the study cohort also limits the generalizability of these findings.

\section{Conclusion}

Based upon current literature, diastolic dysfunction appears to represent an early marker of subclinical cardiovascular disease [4]. There is increasing evidence demonstrating the independent predictive value of diastolic dysfunction for risk of development of diastolic heart failure [4]. Our study suggests that the use of calcium channel blockers may have a protective effect against progression of diastolic dysfunction among African American individuals. Identification of factors that can prevent disease progression is particularly important for African American persons who experience substantial risk of developing complications such as diastolic heart failure.

\section{Acknowledgments}

The project described was supported by the National Center for Advancing Translational Sciences, National Institutes of Health, through Grant UL1TR000050. The content is solely the responsibility of the authors and does not necessarily represent the official views of the NIH.

\section{References}

1. Ferdinand K, Armani A. The management of hypertension in African Americans. Crit Pathw Cardiol. 2007; 6:67-71. [PubMed: 17667868]

2. Garg N, Senthilkumar A, Nusair M, et al. Heart Failure with a normal left ventricular ejection fraction: epidemiology, pathophysiology, diagnosis and management. Am J Med Sci. 2013; 346:129-36. [PubMed: 23503335]

3. Shah S. Heart failure with preserved ejection fraction in African Americans. Ethn Dis. 2012; 22:432-8. [PubMed: 23140073]

4. Aljaroudi W, Thomas J, Rodriguez L, et al. Prognostic Value of diastolic dysfunction: a state of the art review. Cardiol Rev. 2014; 22:79-90. [PubMed: 23707994]

5. Von Lueder T, Krum H. RAAS inhibitors and cardiovascular protection in large scale trials. Cardiovasc Drugs Ther. 2013; 27:171-9. [PubMed: 23224653]

6. James P, Oparil S, Carter B, et al. 2014 evidence-based guideline for the management of high blood pressure in adults: report from the panel members appointed to the Eighth Joint National Committee (JNC-8). JAMA. 2014; 311:507-20. [PubMed: 24352797]

7. Sussman J, Vijan S, Hayward R. Using benefit-based tailored treatment to improve the use of antihypertensive medications. Circulation. 2013; 128:2309-17. [PubMed: 24190955]

8. Prendergast H, Dudley S, Kane J, et al. Progression of left ventricular diastolic dysfunction in ethnic minorities. High Blood Press Cardiovasc Prevention. 2013 Epub ahead of print.

9. Recommendations for Chamber Quantification: A Report from the American Society of Echocardiography's Guidelines and Standards Committee and the Chamber Quantification Writing 
Group, Developed in Conjunction with the European Association of Echocardiography, a Branch of the European Society of Cardiology. J Am Soc Echocardiogr. 2005; 18:1440-63. [PubMed: 16376782]

10. Flack J, Sica D, Bakris G, et al. Management of high blood pressure in blacks: an update of the international society on hypertension in blacks consensus statement. Hypertension. 2010; 56:780800. [PubMed: 20921433]

11. Ozkor M, Rahman A, Murrow J, et al. Differences in vascular nitric oxide and endotheliumderived hyperpolarizing factor bioavailability in blacks and whites. Arterioscler Thromb Vasc Biol. 2014; 34:1320-27. [PubMed: 24675657]

12. Ding Y, Vaziri N. Nifedipine and diltiazem but not verapamil up-regulate endothelial nitric-oxide synthase expression. JPET. 2000; 292:606-9.

13. Ding Y, Vaziri N. Calcium channel blockade enhances nitric oxide synthase expression by cultured endothelial cells. Hypertension. 1998; 32:718-23. [PubMed: 9774369]

14. Tabrizchi R. Amlodipine and endothelial nitric oxide synthase activity. Cardiovasc Res. 2003; 53:807-9. [PubMed: 14553819]

15. Brener S, Ivanc T, Poloszczuk R, et al. Antihypertensive therapy and regression of coronary artery disease: insights from the Comparison of Amlodipine versus Enalapril to Limit Occurrences of Thrombosis(CAMELOT) and Norvasc for Regression of Manifest Atherosclerotic Lesions by Intravascular Sonographic Evaluation (NORMALISE) trials. Am Heart J. 2006; 15:1059-63. [PubMed: 17161053]

16. Wood P, Piran S, Liu P. Diastolic heart failure: progress, treatment challenges, and prevention. Can J Cardiol. 2011; 27:302-10. [PubMed: 21601770]

17. Gradman A, Basile J, Carter B, et al. Combination therapy in hypertension. JASH. 2010; 4:90-8. [PubMed: 20400053]

18. Bruasoulis A, Agarwal V, Tousoulis D, et al. Effects of antihypertensive treatment in patients over 65 years of age: a meta-analysis of randomized controlled studies. Heart. 2014; 100:317-23. [PubMed: 23813846]

19. Joffe S, Phillips R. Treating hypertension in patients with left ventricular dysfunction: hitting the fairway and avoiding the rough. Curr Heart Fail Rep. 2013; 10:157-64. [PubMed: 23563890]

20. Glezeva N, Baugh J. Role of inflammation in the pathogenesis of heart failure with preserved ejection fraction and its potential as a therapeutic target. Heart Fail Rev. 2013 Sep 5. Epub ahead of print.

21. Glezeva N, Baugh J. Role of inflammation in the pathogenesis of heart failure with preserved ejection fraction and its potential as a therapeutic target. Heart Fail Rev. 2013 Sep 5. Epub ahead of print. 


\section{Table 1}

Baseline characteristics of retrospective study cohort $\mathrm{N}=96$. Retrospective study cohort characteristics at baseline

\begin{tabular}{|c|c|}
\hline \multicolumn{2}{|l|}{ Demographics } \\
\hline Age, mean (SD) & $64.2(10.1)$ \\
\hline Female, N (\%) & $75(78.1)$ \\
\hline \multicolumn{2}{|l|}{ Race, N (\%) } \\
\hline African American & $96(100.0)$ \\
\hline Time Followed (years), mean (SD) & $2.6(1.0)$ \\
\hline \multicolumn{2}{|l|}{ Physical measures } \\
\hline BMI, mean (SD) & $31.2(7.1)$ \\
\hline Systolic BP, mean (SD) & $138.0(20.7)$ \\
\hline Diastolic BP, mean (SD) & $75.9(11.9)$ \\
\hline BUN, mean (SD) & $17.9(11.0)$ \\
\hline Creatinine, mean (SD) & $1.4(1.5)$ \\
\hline Hemoglobin, mean $(\mathrm{SD})^{2}$ & $12.6(1.6)$ \\
\hline \multicolumn{2}{|l|}{ Medical history } \\
\hline Smoker, N (\%) & $69(71.9)$ \\
\hline Diabetes, N (\%) & $60(62.5)$ \\
\hline $\mathrm{CAD}, \mathrm{N}(\%)$ & $58(60.4)$ \\
\hline \multicolumn{2}{|l|}{ Medications } \\
\hline Beta Blockers, N (\%) & $35(36.5)$ \\
\hline ACEI, N (\%) & $57(59.4)$ \\
\hline ARB, N (\%) & $65(67.7)$ \\
\hline Calcium Channel Blocker, N (\%) & $59(61.5)$ \\
\hline Diuretics, N (\%) & $44(45.8)$ \\
\hline \multicolumn{2}{|l|}{ Diastolic descriptors } \\
\hline \multicolumn{2}{|l|}{ LA size, $\mathrm{N}(\%)$} \\
\hline Normal & $55(57.3)$ \\
\hline Mild & $31(32.3)$ \\
\hline Moderate & $10(10.4)$ \\
\hline \multicolumn{2}{|l|}{ Diastolic dysfunction, $\mathrm{N}(\%)$} \\
\hline Grade 0 & $2(2.1)$ \\
\hline Grade 1 & $85(88.5)$ \\
\hline Grade 2 & $6(6.3)$ \\
\hline Grade 3 & $3(93.1)$ \\
\hline LVH, N (\%) & $40(41.7)$ \\
\hline \multicolumn{2}{|l|}{ LVH Degree, N (\%) } \\
\hline Normal & $35(36.5)$ \\
\hline Mild & $40(41.7)$ \\
\hline Moderate & $18(18.8)$ \\
\hline Severe & $3(3.1)$ \\
\hline
\end{tabular}

High Blood Press Cardiovasc Prev. Author manuscript; available in PMC 2016 January 14. 
Table 2

Risk Factors Evaluated by Progression Status in an African American Cohort. Univariate Risk Factor Analysis by diastolic dysfunction progression in an African American Cohort

\begin{tabular}{|c|c|c|c|}
\hline & $\begin{array}{l}\text { Progression } \\
\mathrm{N}=22\end{array}$ & $\begin{array}{c}\text { No Progression } \\
\quad \mathbf{N}=74\end{array}$ & p-value \\
\hline \multicolumn{4}{|l|}{ Demographics } \\
\hline Age, mean (SD) & $59.9(11.3)$ & $65.5(9.4)$ & 0.0212 \\
\hline Age $\geq 65, N(\%)$ & $7(31.8)$ & $42(56.8)$ & 0.0399 \\
\hline Female, N (\%) & $6(27.3)$ & $15(20.3)$ & 0.4855 \\
\hline Follow-up time (years), mean (SD) & $3.1(2.6)$ & $2.4(2.2)$ & 0.0041 \\
\hline \multicolumn{4}{|l|}{ Physical measures } \\
\hline BMI, mean (SD) & $32.1(6.3)$ & $30.9(7.3)$ & 0.5070 \\
\hline Obese, $\mathrm{N}(\%)$ & $14(63.6)$ & $36(48.7)$ & 0.2167 \\
\hline Overweight or Obese, N (\%) & $18(81.8)$ & $56(75.7)$ & 0.5473 \\
\hline Systolic BP, mean (SD) & $138.7(21.1)$ & $137.7(20.7)$ & 0.8461 \\
\hline Diastolic BP, mean (SD) & $76.2(11.8)$ & $75.8(12.0)$ & 0.8860 \\
\hline BUN, mean (SD) & $15.5(7.7)$ & $18.7(11.8)$ & 0.1375 \\
\hline Creatinine, mean (SD) & $1.4(1.8)$ & $1.4(1.4)$ & 0.8776 \\
\hline Hemoglobin, mean (SD) & $12.4(1.1)$ & $12.7(1.7)$ & 0.2894 \\
\hline \multicolumn{4}{|l|}{ Medication use } \\
\hline Beta Blockers, N (\%) & $9(40.9)$ & $26(35.1)$ & 0.6213 \\
\hline ACEI, N (\%) & $15(68.2)$ & $42(56.8)$ & 0.3381 \\
\hline ARB, N (\%) & $14(63.6)$ & $51(68.9)$ & 0.6418 \\
\hline Calcium Channel Blockers, N (\%) & $10(45.5)$ & $49(66.2)$ & 0.0790 \\
\hline Diuretics, N(\%) & $7(31.8)$ & $37(50.0)$ & 0.1329 \\
\hline \multicolumn{4}{|l|}{ Medical history } \\
\hline Smoker, N (\%) & $16(72.7)$ & $53(71.6)$ & 0.9193 \\
\hline Diabetes, N (\%) & $13(59.1)$ & $47(63.5)$ & 0.7068 \\
\hline \multicolumn{4}{|l|}{ Echocardiogram parameters } \\
\hline LA Size Normal, N (\%) & $13(59.1)$ & $42(56.8)$ & 0.8459 \\
\hline Left Ventricular Hypertrophy, N (\%) & $12(54.5)$ & $28(37.8)$ & 0.1628 \\
\hline
\end{tabular}


Table 3

Multivariate Logistic Regression for covariates and diastolic dysfunction progression in an African American Cohort. Multivariate Logistic Regression involving hypertension medication classification, age, BMI and diastolic dysfunction progression in an African American Cohort

\begin{tabular}{lllll}
\hline Effect & p value & Odds Ratio reference & Value & 95 \% Wald CI \\
\hline Baseline ACE-I & 0.0589 & Users vs. non-users & 3.42 & $(0.96,12.27)$ \\
Baseline Age & 0.0548 & Age $ð 5$ vs. <65 & 0.32 & $(0.10,1.02)$ \\
Baseline CCB & 0.0329 & Users vs. non-users & 0.28 & $(0.09,0.90)$ \\
Baseline Diuretics & 0.1456 & Users vs. non-users & 0.42 & $(0.13,1.35)$ \\
Baseline BMI & 0.1292 & Obese vs. non-obese & 2.43 & $(0.77,7.61)$ \\
Baseline LVH & 0.1873 & LVH vs. no LVH & 2.14 & $(0.69,6.65)$ \\
\hline
\end{tabular}

